\title{
Modelos de front end da inovação: similaridades, diferenças e perspectivas de pesquisa
}

\author{
Pierry Teza ${ }^{a}$, Gertrudes Dandolinia, João Artur de Souzaa, Viviane Brandão Migueza, \\ Roberto Fabiano Fernandes ${ }^{\mathrm{b}}$, Paulo Augusto Cauchick Miguel ${ }^{\mathrm{a} *}$ \\ a*Universidade Federal de Santa Catarina, Florianópolis, SC, Brasil, paulo.cauchick@ufsc.br \\ bUniversidade do Estado de Santa Catarina, Florianópolis, SC, Brasil
}

\begin{abstract}
Resumo
0 front end da inovação é o primeiro subprocesso do processo da inovação. Esse subprocesso reúne as atividades realizadas antes da proposição e aprovação de um conceito a ser desenvolvido e implementado. Uma vez que as organizações podem se beneficiar substancialmente da otimização e melhoria do front end da inovação, e que esse ainda tem recebido pouca atenção, tanto da academia quanto das organizações, justifica-se a importância de estudos que busquem a compreensão e a organização desse corpo da literatura, auxiliando assim na evolução dos conceitos teóricos, bem como o preenchimento das lacunas de pesquisa ainda existentes. Nesse sentido, este artigo relata os resultados de um estudo cujo objetivo foi, a partir de levantamento e análise da literatura relativa ao front end da inovação e, em especial, dos modelos desse subprocesso, propor um conjunto de pressupostos como base para o desenvolvimento teórico e empírico do tema. Com o levantamento, realizado em quatro bases de dados eletrônicas, foram identificados 268 trabalhos, dos quais oito apresentam modelos de front end da inovação considerados relevantes para análise. Esses modelos são comparados com base em alguns critérios de análise de conteúdo dos artigos. A partir dessa análise, também são sugeridas perspectivas de pesquisa, bem como pontos que necessitam de aprofundamento teórico são identificados.
\end{abstract}

Palavras-chave

lnovação. Front end da inovação. Gestão da inovação. Revisão de literatura.

\section{Introdução}

0 estudo da inovação ganhou notoriedade a partir da década de 1980, quando as organizações começaram a perceber que a capacidade de inovar afeta significativamente o futuro do negócio. A inovação representa o processo de renovação principal em qualquer organização, haja vista que as mudanças que as organizações oferecem, por meio de seus produtos, e as formas com que elas criam e fornecem essas ofertas, diz respeito a sua sobrevivência e perspectiva de crescimento (Bessant et al., 2005). Existem variados pontos de vista e conceitos referentes à inovação (Baregheh et al., 2009; Crossan \& Apaydin, 2010; Quintane et al., 2011). Baregheh et al. (2009), buscando uma definição que representasse a sua essência, afirmam que a inovação é o processo de várias etapas através do qual as organizações transformam ideias em produtos, serviços ou processos novos ou melhorados, a fim de avançar, competir e diferenciar-se com sucesso em seu mercado.

Ao longo dos anos, pesquisadores e profissionais em geral têm buscado formas de melhorar o processo de inovação. Nesse sentido, diversos trabalhos (e.g. Nobelius \& Trygg (2002); Perttula (2004); Backman et al. (2007); Williams et al. (2007); Aagaard \& Gertsen (2011)) têm verificado que as decisões tomadas no início do processo tendem a direcionar as demais decisões e que elas têm o maior potencial para melhorar a inovação com um menor esforço. Assim, uma melhoria nesse início tem um impacto positivo no processo como um todo (Reid \& Brentani, 2004). Smith \& Reinertsen (1991) propuseram uma divisão do processo de inovação em três subprocessos: fuzzy front end (denominado 
neste trabalho de "front end da inovação" - FEI); desenvolvimento de novos produtos (chamado neste trabalho de "desenvolvimento"); comercialização (denominado neste trabalho de "implementação"). 0 uso de uma terminologia alternativa fundamenta-se no fato de que nem toda inovação é um produto e nem toda inovação vai ser comercializada. Por exemplo, inovações decorrentes de novos métodos de marketing e organizacionais. Nesse sentido, objetiva-se obter uma visão mais abrangente do front end da inovação.

Na proposta apresentada por Smith \& Reinertsen (1991) é dada ênfase ao início do processo da inovação sem com isso se negligenciar o restante desse processo. Analisando a literatura sobre inovação, verifica-se que dos três subprocessos apresentados, muito se avançou em relação ao desenvolvimento e à implementação, principalmente no contexto do projeto e oferta de produtos (bens). Porém, os estudos relativos ao front end da inovação só recentemente têm recebido a atenção devida, tanto no âmbito empresarial quanto no da academia (Aagaard \& Gertsen, 2011). lsso parece contraditório, uma vez que o FEl fundamentalmente determina o posterior sucesso da inovação (Koen et al., 2001).

Este trabalho parte da premissa de que a gestão do FEl tende a ser eficaz no momento em que se dispõe de um processo devidamente estruturado, haja vista que estudos indicam que isso tende a trazer vantagens para a organização (Flint, 2002; Hüsig \& Kohn, 2003). Porém, uma vez que não se pode dispor de um processo adequado a todas as situações (Nobelius \& Trygg, 2002), deve-se investir na obtenção de conhecimentos que possam auxiliar na estruturação de processos específicos para contextos específicos. Para isso, identificou-se a necessidade de organização dos conhecimentos presentes na literatura sobre FEl, mais especificamente sobre os modelos desse subprocesso, uma vez que não foram encontrados trabalhos que propusessem uma análise nesse sentido. Assim, este artigo relata os resultados de um estudo cujo objetivo foi, a partir de levantamento e análise da literatura relativa ao FEl, e em especial aos modelos desse subprocesso, propor um conjunto de pressupostos como base para o desenvolvimento teórico e empírico do tema. Adicionalmente, sugere perspectivas de pesquisas que podem contribuir para o avanço do conhecimento em relação ao tema.

\section{Front end da inovação: aspectos gerais}

Existem variados pontos de vista e conceitos referentes à inovação (Baregheh et al., 2009; Crossan \& Apaydin, 2010; Quintane et al., 2011).
Baregheh et al. (2009, p. 1334) afirmam, com base na análise de definições da literatura, que a "[...] inovação é o processo de várias etapas através das quais as organizações transformam ideias em produtos novos/melhorados, serviços ou processos, a fim de avançar, competir e diferenciar-se com sucesso em seu mercado". A inovação pode ver vista como um processo (e.g. na definição de Baregheh et al. (2009)) ou como o resultado desse processo (Organização de Cooperação e Desenvolvimento Econômico, 2005): inovação em produtos, em processos, em métodos de marketing, ou métodos organizacionais. Pode ser também vista na perspectiva da intensidade de resultado (Garcia \& Calantone, 2002; Reid \& Brentani, 2004): inovação radical ou incremental. Mesmo dispondo dessas tipologias, deve-se considerar que existem algumas situações em que há certa dificuldade para identificar o tipo de inovação, já que muitas vezes não existe uma linha divisória clara entre um tipo e outro de inovação (Tidd et al., 2008).

0 presente artigo tem como foco o processo de inovação cujos estudos derivaram daqueles centrados no desenvolvimento de produtos, com foco em bens de consumo. Nesse sentido, é importante estabelecer uma perspectiva histórica das abordagens relacionadas à inovação. Considera-se que Rothwell (1994) traduz essa evolução de forma mais fidedigna. Assim, sob uma perspectiva histórica, essas abordagens podem ser divididas em cinco gerações, conforme ilustra a Figura 1. A Tabela 1 sintetiza essas gerações.

Com respeito aos modelos de quinta geração, destacam-se duas abordagens recorrentes na literatura: o Funil de Desenvolvimento (Clark \& Wheelwirght, 1993) e o Stage-Gate (Cooper, 1993). Essas abordagens têm sido fortemente atreladas à literatura de desenvolvimento de novos produtos, porém aqui serão tratadas como modelos de processos de inovação.

0 modelo Funil de Desenvolvimento (Figura 2), desenvolvido por Clark \& Wheelwright (1993), considera que o processo de inovação inicia-se pelo planejamento de um conjunto de projetos (portfólio). Por meio de um processo com fases e avaliações, a organização vai mantendo os projetos com maior probabilidade de sucesso até o mercado. Observa-se também que essa abordagem já leva em consideração uma fase para desenvolvimento de conceitos (Fase 1), ideia central para o front end da inovação. Já o modelo Stage-Gate, com cinco estágios e gates (Cooper, 1993), sofreu evoluções importantes ao longo do tempo (e.g. Cooper \& Edgett (2008); Cooper (2011)), mas mantendo sua estrutura inicial baseada no conceito de estágios 


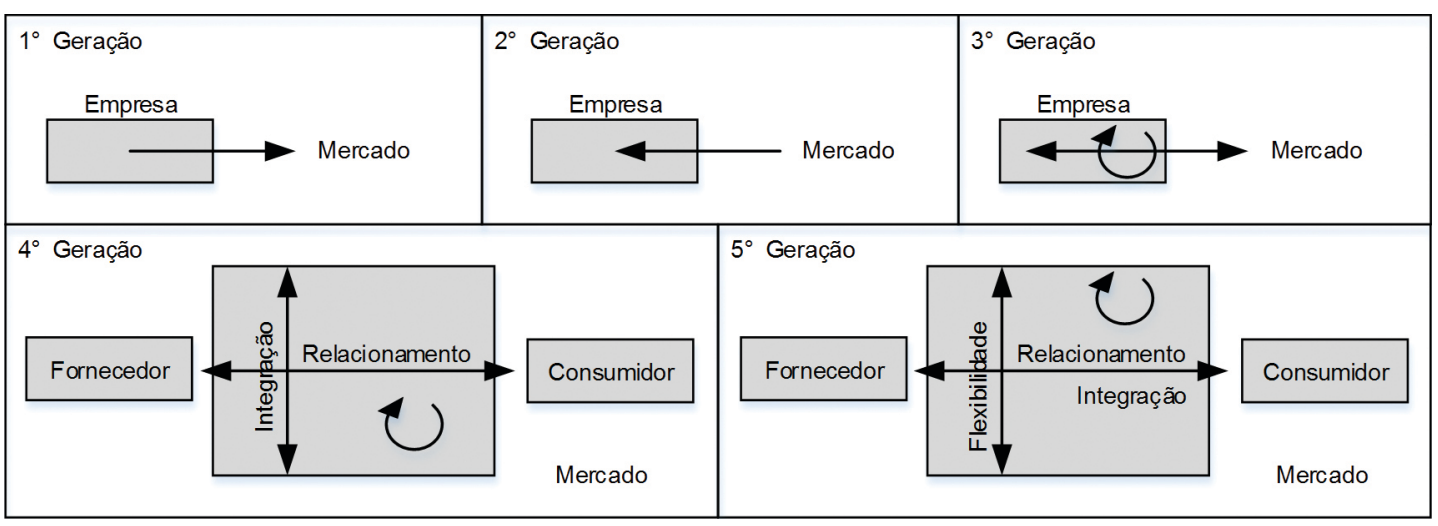

Figura 1. Cinco gerações dos modelos de inovação. Fonte: Cagnazzo et al. (2008), baseado em Rothwell (1994).

Tabela 1. Síntese das abordagens relacionadas à inovação.

\begin{tabular}{|c|c|c|}
\hline Geração & Período & Caracteristicas do processo \\
\hline $1^{a}$ & $1950-60$ & Linear e apoiado sobre pesquisa e desenvolvimento em um sistema empurrado pela tecnologia (technology-push) \\
\hline $2^{\mathrm{a}}$ & $1960-70$ & Linear e orientado pelo mercado, ou seja, a inovação é puxada pelo mercado (market-pull) \\
\hline $3^{\mathrm{a}}$ & $1970-80$ & $\begin{array}{l}\text { Interação entre diversas atividades de inovação (pesquisa, design, desenvolvimento, prototipagem e manufatura), } \\
\text { guiado tanto por fatores tecnológicos quanto mercadológicos }\end{array}$ \\
\hline $4^{\mathrm{a}}$ & 1980-90 & Maior integração entre atividades e execução paralela dos processos \\
\hline $5^{\mathrm{a}}$ & 1990-atual & $\begin{array}{l}\text { Maior preocupação na busca da eficiência no processo (e.g. custo e tempo de desenvolvimento), centrado na } \\
\text { interação entre as etapas e as atividades do processo }\end{array}$ \\
\hline
\end{tabular}

Fonte: Construido pelos autores com base em Rothwell (1994).

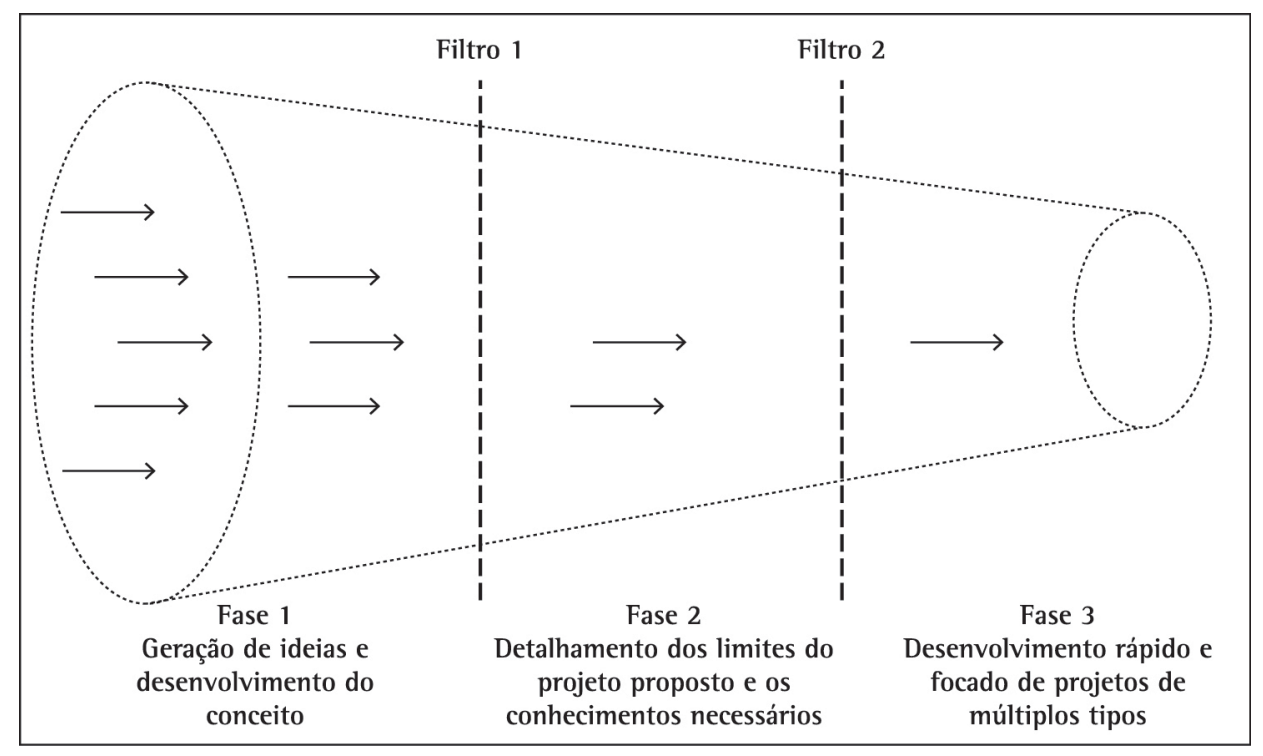

Figura 2. Funil de Desenvolvimento (Clark \& Wheelwright, 1993).

definidos e pontos de decisão representados por gates (Figura 3). 0 processo começa com uma ideia, que vai se desenvolvendo ao longo desse processo. Em cada um dos gates ocorre uma decisão de continuar ou interromper o projeto. 0 Stage-Gate destina-se a canalizar os projetos de desenvolvimento de novos produtos, eliminando aqueles que não têm potencial antes que acumulem custos elevados, utilizando muitos recursos.
Nos últimos anos tem-se verificado um crescente interesse pela questão dos serviços (exemplos podem ser vistos em Alam (2006), Hennala et al. (2011), Zomerdijk \& Voss (2011)), tendo surgido inclusive o termo "desenvolvimento de novos serviços" (NSD ou New Service Development). Também verifica-se o interesse por inovações em processos (por exemplo, em Kurkkio et al. (2011)) e em métodos organizacionais (por exemplo, em Günzel \& Holm 
(2013)). Nesse sentido, supõe-se uma tendência no estudo de outros tipos de inovação seguida da necessidade de uma visão holística do processo, uma vez que as atividades realizadas no início do processo de inovação - front end da inovação - podem dar origem a quaisquer dos tipos de inovação: produtos (bens ou serviços); processos; métodos de marketing; métodos organizacionais.

\subsection{Front end da inovação}

Uma mudança relevante na forma de ver o processo de inovação foi realizada por Smith \& Reinertsen em 1991, os quais, ainda com foco no desenvolvimento de produtos, dividiram o processo de desenvolvimento em três subprocessos - fuzzy front end (FFE), desenvolvimento de novos produtos e comercialização - e enfatizaram o primeiro. Esse subprocesso inicial representa as atividades e o tempo até a proposição e aprovação de um conceito de produto. 0 termo "fuzzy" indica o quanto esse subprocesso pode ser caótico, imprevisível e incerto e seu uso, de acordo com Koen et al. (2001), pode implicar que essa parte do processo da inovação é nebulosa e isso pode resultar em falta de responsabilidade e dificuldade na identificação de quem é responsável por gerenciar as atividades. Nesse sentido, esses autores recomendam o termo "front end" da inovação - terminologia que decidiu-se adotar neste artigo, como já mencionado. Porém ainda não existe na literatura um consenso sobre essa terminologia, como pode ser constatado na Tabela 2 .

Deve-se observar também em relação à terminologia que, antes da contribuição de Smith \& Reinertsen (1991), esse subprocesso já era estudado (como, por exemplo, em um dos trabalhos seminais de Cooper (1988)), porém com outros termos. Analisando o Funil de Inovação e o Stage-Gate, observa-se que o front end da inovação nada mais é do que o início desses processos. Koen et al. (2001) afirmam que o $\mathrm{FEl}$ envolve as atividades que ocorrem antes do desenvolvimento de produtos. Khurana \& Rosenthal (1998) complementam afirmando que esse subprocesso inclui a formulação do produto e da estratégia de comunicação, a identificação de oportunidades e a avaliação, a geração de ideias, a definição de produto, o planejamento de projetos e as revisões executivas. Uma forma de entender melhor o conceito e a natureza do front end da inovação é compará-lo ao subprocesso de Desenvolvimento de Novos Produtos (DNP), conforme mostra a Tabela 3.

Observa-se, pela análise da Tabela 3 , a característica difusa e pouco estruturada do FEl. Nesse sentido, justificam-se as dificuldades encontradas tanto pela academia quanto pelas organizações para a proposição e implementação de

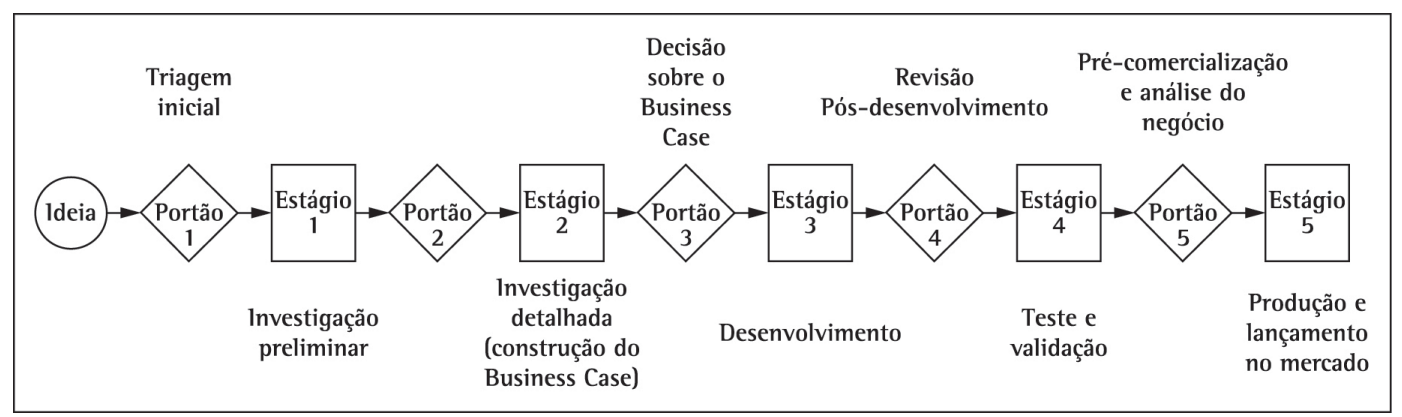

Figura 3. 0 processo de Stage-Gate (Cooper, 1993).

Tabela 2. Termos relativos ao front end da inovação.

\begin{tabular}{|c|c|}
\hline Termo & Literatura \\
\hline fuzzy front end & $\begin{array}{l}\text { Khurana \& Rosenthal (1997), Reinertsen (1999), Montoya-Weiss \& O’Driscoll (2000), Flint (2002), Kim \& } \\
\text { Wilemon (2002), Alam (2006), Magnusson (2009), Frishammar et al. (2011), Kurkkio (2011), Brentani \& Reid } \\
\text { (2012) }\end{array}$ \\
\hline front end of innovation & $\begin{array}{l}\text { Koen et al. (2001), Poskela \& Martinsuo (2009), Martinsuo \& Poskela (2011), Bertels et al. (2011), Hannola \& } \\
\text { Ovaska (2011) }\end{array}$ \\
\hline front end process & Nobelius \& Trygg (2002) \\
\hline $\begin{array}{l}\text { front end of new product } \\
\text { development }\end{array}$ & Oliveira \& Rozenfeld (2010) \\
\hline early phases of innovation & Flanagin (2000), Brolø (2009), Muhdi et al. (2011) \\
\hline pre-development & Cooper (1988), Toledo et al. (2008), Meijer et al. (2007), Hammedi et al. (2011) \\
\hline
\end{tabular}

Fonte: Construída pelos autores com base na literatura. 
mecanismos que possam auxiliar a tomada de decisão nesse início do processo de inovação. Por outro lado, as dificuldades de estruturação do FEl representam oportunidades para pesquisadores e gestores.

\subsection{Síntese dos resultados para o FEl}

A Tabela 4 ilustra, de modo resumido, diferentes características para o FEl indicadas na literatura analisada.

Com base na análise realizada das publicações, considerou-se como saída do FEl um conceito, podendo ele ser de um novo ou melhorado produto (bem ou serviço), processo, método de marketing ou método organizacional. Porém considera-se que esse conceito pode ser diferente em função da organização e de seu setor de atuação, ou seja, a forma final do conceito é dependente do contexto, podendo assumir quaisquer das formas citadas anteriormente como resultados do FEl.

\section{Procedimentos metodológicos}

A estrutura metodológica do trabalho foi dividida em seis etapas: (i) identificação das palavras-chave; (ii) busca em bases de dados selecionadas; (iii) seleção das publicações; (iv) inclusão de outras publicações relevantes; (v) identificação de modelos de FEl; e (vi) análise dos modelos. Essas etapas estão detalhadas na sequência. (i) Identificação das palavras-chave: Foram identificadas por meio de leitura exploratória, das quais obtiveram-se: fuzzy front end; front end of innovation; front end process; front end of new product development; early phases of innovation; early stages of innovation; pre-development. Observando-se os termos, verifica-se que os quatro primeiros têm em comum o termo "front end". Assim, para as buscas utilizaram-se os seguintes termos combinados com "innovation": "front end", "early phases of innovation", "early stages of innovation" e "pre-development".

ii) Buscas nas bases de dados: As buscas foram realizadas em quatro bases de dados, em março de 2013: Scopus, Web of Knowledge, EBSCO, e Engineering Village. A escolha dessas bases deu-se por elas serem, na área de gestão de negócios, reconhecidas pela qualidade das publicações armazenadas. Optou-se pela utilização apenas de artigos de periódicos, uma vez que já estão avaliados pelos pares e assim constituem fonte com maior reputação para análise. As pesquisas foram realizadas nos títulos, resumos e palavras-chaves dos artigos, obtendo-se um total de 1.032 artigos.

iii) Seleção das publicações: 0 software EndNote ${ }^{\circledR}$ foi utilizado e as publicações foram importadas para ele. Também foram eliminadas as publicações duplicadas entre as bases. Em uma primeira leitura dos títulos e dos resumos, foram retirados os artigos que não eram relacionados ao tema; por exemplo, artigos da área de eletrônica, que estavam incluídos por possuírem os termos "front end" e "innovation"

Tabela 3. Comparação entre o FEl e o DNP.

\begin{tabular}{|c|c|c|}
\hline Dimensão & Fuzzy front end" & Desenvolvimento de novos produtos \\
\hline Natureza do trabalho & $\begin{array}{l}\text { Experimental, muitas vezes caótico; difícil de planejar; } \\
\text { momentos de eureka }\end{array}$ & $\begin{array}{l}\text { Estruturado, disciplinado e orientado por um plano de } \\
\text { projeto }\end{array}$ \\
\hline Data de comercialização & Imprevisível & Definível \\
\hline Financiamento & $\begin{array}{l}\text { Variável; nas fases iniciais, muitos projetos poderão } \\
\text { ser cancelados, enquanto outros vão precisar de } \\
\text { financiamento para prosseguir }\end{array}$ & Orçado \\
\hline Expectativas de receita & $\begin{array}{l}\text { Muitas vezes incerto: às vezes feito com uma grande } \\
\text { dose de especulação }\end{array}$ & $\begin{array}{l}\text { Acreditável e com aumento da certeza, análise e } \\
\text { documentação à medida que a data de lançamento do } \\
\text { produto se aproxima }\end{array}$ \\
\hline Tipo de atividades & $\begin{array}{l}\text { Tanto individual quanto em equipe, em áreas para } \\
\text { minimizar os riscos e otimizar o potencial }\end{array}$ & $\begin{array}{l}\text { Equipes multifuncionais de produto e/ou de } \\
\text { desenvolvimento do processo }\end{array}$ \\
\hline
\end{tabular}

*Terminologia originalmente utilizada por Koen et al. (2001). Fonte: Koen et al. (2001).

Tabela 4. Resultados para o FEl.

\begin{tabular}{ll}
\hline \multicolumn{1}{c}{ Característica } & \multicolumn{1}{c}{ Literatura } \\
\hline Conceitos de produtos & Cooper (1993), Koen et al. (2001), Murphy \& Kumar (1997), Whitney (2007) \\
Seleção da oportunidade certa & Kim \& Wilemon (2002) \\
Geração de propriedade intelectual & Koen et al. (2001) \\
ldeias e/ou soluções & Flynn et al. (2003) \\
Projetos preliminares & Boeddrich (2004), Sandmeier (2009), Kurkkio et al. (2011) \\
Plano de negócios & Crawford et al. (2006) \\
\hline
\end{tabular}

Fonte: Construído pelos autores com base na literatura. 
utilizados também nessa área, mas com outro significado. Finalmente, foram eliminados aqueles artigos que tratavam superficialmente do tema. Esse último passo foi realizado por três autores, com base na leitura dos título e resumo de cada publicação e, caso necessário, na leitura do artigo completo. Foram então retirados todos os artigos que receberam duas indicações de eliminação. Desse processo restaram 256 artigos, dos quais 173 estavam disponíveis para download.

iv) Inclusão de outras publicações relevantes: De forma a evitar que artigos importantes não fossem analisados em função de não estarem presentes em nenhuma das bases utilizadas, as referências das 173 publicações disponíveis em texto completo foram analisadas com o objetivo de identificar artigos relevantes. Nesse passo foram selecionados mais 12 artigos relacionados ao tema (sete deles disponíveis na íntegra). Esses artigos foram adicionados ao portfólio final, que resultou, assim, em 268 publicações (portfólio 1), sendo 180 disponíveis na íntegra.

v) Busca por modelos: A partir do portfólio 1 iniciou-se a análise dos artigos procurando identificar aqueles que apresentavam algum tipo de modelo de front end da inovação, bem como os artigos que auxiliassem a análise dos modelos encontrados. Considerou-se como modelo toda "[...] representação de objetos ou situações reais" (Anderson et al., 1991). Ou seja, os modelos deveriam conceitualmente representar o front end da inovação, seja a partir de uma situação real (caráter descritivo) ou a partir de uma situação desejada (caráter prescritivo - ou normativo). Ao final dessa busca foram identificados oito modelos relevantes para o estudo proposto (portfólio 2).

vi) Análise dos modelos: Inicialmente buscou-se nos modelos as seguintes informações: contexto sob o qual o modelo foi concebido; origem (teórica ou empírica); fluxo do processo (predominantemente linear ou interativo); elementos considerados (oportunidades, ideias e/ou conceitos); atividades indicadas. Uma vez levantadas essas informações, procurou-se analisá-las com vistas a obter diferenças e similaridades entre os modelos, de modo a gerar inferências que pudessem conduzir ao alcance dos objetivos do estudo. Apesar de a análise focar prioritariamente nos modelos de FEl (portfólio 2), o conjunto total de artigos relativos ao tema (portfólio 1) foi utilizado como base teórica para a análise dos modelos, participando, assim, nos estabelecimentos dos pressupostos e no desenvolvimento das perspectivas de pesquisa.

Com base nas etapas indicadas, descrevem-se a seguir os resultados do trabalho, iniciando-se por uma síntese dos modelos encontrados.

\section{Front end da inovação: uma análise dos modelos}

Estudos verificados nessa revisão de literatura indicam que um melhor resultado para o processo de inovação obtém-se com um FEl estruturado (Flint, 2002; Hüsig \& Kohn, 2003), ou seja, composto por um conjunto de atividades e relacionamentos predefinidos. Conforme alegam Brem \& Voigt (2009), contextos difíceis de prever e planejar, como o FEI, requerem processos mais estruturados e disciplinados para obtenção de novas ideias.

Hüsig \& Kohn (2003) afirmam que, no FEl, um processo de estágios e gates (pontos de decisão) que leva a um conceito de produto detalhado, estruturado e claro é composto por atividades realizadas por uma equipe cross-funcional apoiada pela alta direção e com recursos suficientes. Atividades que geralmente estão associadas ao FEl são a identificação e análise de oportunidades, geração e seleção de ideias e desenvolvimento de conceitos (Koen et al., 2001; Whitney, 2007; Aagaard \& Gertsen, 2011). A equipe que realiza essas atividades deve ser o centro de uma rede composta por ligações entre os departamentos da empresa, bem como com o ambiente externo da organização (Hüsig \& Kohn, 2003). Considera-se nesse sentido que um FEl estruturado define fases, entradas, saídas, bem como pontos de decisão e as informações usadas neles (Paasi et al., 2007).

Entretanto, Backman et al. (2007) verificaram em seu estudo na indústria automobilística que um processo estruturado, com muitas regras e requisitos, pode limitar conceitos inovadores que se afastam daqueles mais facilmente reconhecíveis em vez de investigar suas potenciais oportunidades. De acordo com Reid \& Brentani (2004), inovações radicais não correspondem ao resultado de processos organizacionais explícitos e estruturados, como no caso de inovações incrementais, mas sim ao de um complexo fenômeno de compartilhamento de informações que ocorre da base para o topo da pirâmide organizacional, principalmente no FEl.

Assim, a inovação deve ser intencional e apoiada pelos processos de uma organização (Perttula, 2004), considerando aspectos dos ambientes interno e externo e sem deixar também que oportunidades e ideias importantes, que fujam ao paradigma vigente, percam-se. Atingir um balanço entre formalismo e criatividade é um desafio para as organizações (Salmela et al., 2013). Nesse sentido, não há um processo único, um modelo de FEl adequado para todas as situações, mas sim o tipo de projeto e a situação geral da empresa devem influenciar a organização e estruturação desse subprocesso (Nobelius \& Trygg, 2002; Salmela et al., 2013). Assim, 
reforça-se a intenção de busca por mecanismos que possam auxiliar gestores a tomar decisões em relação à estruturação do $\mathrm{FEl}$ em contextos específicos.

A Tabela 5 apresenta uma síntese comparativa entre os modelos identificados, tendo como objetivo buscar similaridades e diferenças, bem como contribuições para a proposta do presente trabalho.

Com relação à origem dos modelos estudados, evidenciou-se que quatro deles têm origem empírica e os outros quatro, teórica. Não se pode deixar de mencionar que os modelos teóricos, de alguma forma, incorporam a experiência de seus autores, como é o caso de Whitney (2007), cuja experiência em organizações vinculadas a Pesquisa \& Desenvolvimento oferece um modelo com foco nesse tipo de organização. A escassez de tentativas de representação do front end da inovação, sejam elas por meio de proposições teóricas, sejam por estudos empíricos, evidencia o pouco desenvolvimento dos estudos relacionados ao tema. Nesse sentido, a análise dos modelos mostra que eles ainda carecem de aprofundamento para que se possam criar outros modelos operacionalizáveis. Justifica-se a afirmação pelo fato de os modelos, de modo geral, apresentarem o procedimento do processo, bem como suas atividades, porém, de serem escassos em relação às técnicas e ferramentas que podem ser utilizadas, bem como em relação à inter-relação entre elas. Exceção se faz ao modelo de Whitney (2007), que procura apresentar um conjunto de ferramentas e técnicas a serem utilizadas nas atividades propostas por seu modelo. Porém, conforme o próprio autor menciona, muitas ferramentas e técnicas se sobrepõem em diversas atividades e a questão da efetividade delas não foi abordada no trabalho. De fato, tanto a escolha de técnicas e ferramentas para o FEl quanto o estudo da efetividade delas já começam a ser foco de algumas pesquisas (por exemplo, Achiche et al. (2013)), entretanto esse ainda é um campo que necessita de desenvolvimentos teóricos e empíricos.

Observou-se também a predominância de modelos focados em desenvolvimento de bens em oposição a modelos de relacionados aos demais tipos de inovação (serviços, processos, métodos de marketing, métodos organizacionais). Inovação em serviços, por exemplo, tem emergido como um imperativo estratégico para muitas empresas e tem atraído a atenção de pesquisadores e profissionais (Alam, 2006), abrindo novas possibilidades de pesquisa. Destaca-se, nesse sentido, o modelo apresentado por Brem \& Voigt (2009), decorrente de um estudo empírico no setor de software em que os autores abordam a integração entre mercado e tecnologia. Em relação à inovação em processo, destaca-se o modelo apresentado por Kurkkio et al.
(2011), derivado de um estudo multicaso em inovações de processo.

No presente estudo não foram identificados trabalhos que façam menção a modelos de front end para desenvolvimento de novas estratégias de marketing ou métodos organizacionais. De fato, pouca pesquisa tem focado em outros tipos de inovação que não em bens, tais como as inovações em processo, em marketing e em métodos organizacionais, que podem ter implicações relevantes para as organizações (Artto et al., 2011; Günzel \& Holm, 2013). Assim, observam-se argumentos em consonância ao que foi discutido anteriormente: a escassez de desenvolvimento da teoria relativa ao processo do FEl.

De um modo geral, três elementos são recorrentes entre os modelos: oportunidades; ideias; conceitos. As definições adotadas nesta pesquisa para esses elementos são (Koen et al., 2002):

- Ideia é a forma mais embrionária de um novo produto (bem ou serviço);

- Oportunidade corresponde a uma lacuna de negócio ou de cunho tecnológico, que uma empresa ou indivíduo percebe que existe entre a situação atual e um futuro imaginado, a fim de obter vantagem competitiva, responder a uma ameaça, resolver um problema, ou melhorar uma situação;

- Conceito tem uma forma definida, inclusive uma descrição escrita e visual, que considera características e benefícios aos clientes, combinada com um amplo conhecimento da tecnologia necessária.

Complementando a definição de ideia, uma vez que o resultado do FEl pode ser o conceito para uma inovação em produto, processo, marketing ou organizacional, considera-se assim que uma ideia é a forma embrionária não apenas de um novo produto, mas também de um novo processo, método de marketing ou método organizacional. Ainda é importante ressaltar que alguns modelos não apresentam o elemento oportunidades ou ideias. lsso significa que alguns modelos não diferenciam "ideias" de "oportunidades". Observando as definições apresentadas, fica evidente que ideias são diferentes de oportunidades, porém não foi encontrado nenhum estudo que avaliasse o quão diferente esses dois elementos são ou se são diferentes em todos os contextos.

Os modelos, então, para os elementos apresentados, enumeram atividades realizadas sobre esses elementos, tais como: geração, enriquecimento e seleção de ideias, identificação e seleção de oportunidades, entre outras. Ressalta-se uma dificuldade encontrada na análise dos modelos: a 


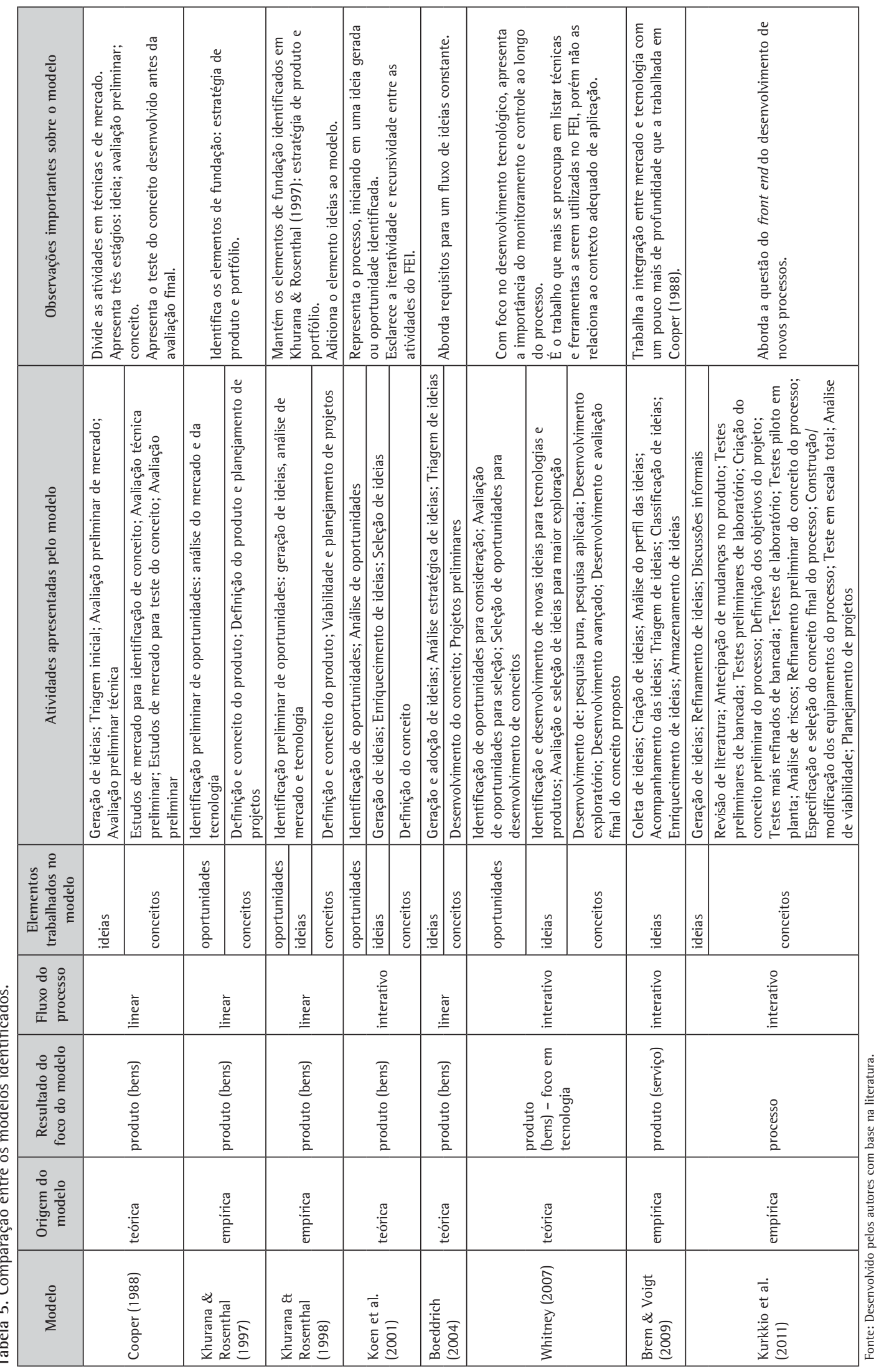


falta de uma estrutura teórica que pudesse auxiliar essa análise e, em um sentido mais pragmático, pudesse ajudar a operacionalização dos modelos. Nesse sentido, entende-se que cada elemento principal deve ser gerido, ou seja, contemplado com um procedimento. Assim, pode-se estabelecer que um macroprocedimento, que compreenderia a gestão do FEl, poderia desdobrar-se em procedimentos mais detalhados, que corresponderiam à gestão de cada um dos elementos principais, cuja nomenclatura já vem sendo utilizada na literatura de inovação e desenvolvimento de novos produtos: gestão de oportunidades, gestão de ideias e gestão de conceitos.

Apesar de os modelos analisados apresentarem as atividades relativas aos elementos principais sem explicitarem a interação entre elas, observou-se que, de um modo geral, os trabalhos salientam a relação interativa entre essas atividades. Mesmo que alguns modelos não tenham sido classificados como interativos, por esse movimento não estar devidamente explicado no trabalho, a maioria deles reconhece que as atividades influenciam-se mutuamente e não há uma sequência exata de execução. Por exemplo, o caso da geração de ideias e da identificação de oportunidades, como explicitado por Vandenbosch et al. (2006) e Koen et al. (2001), em que uma oportunidade pode levar a uma ou mais ideias, mas também uma ideia pode levar à necessidade de uma ou mais oportunidades para ser de execução viável. Nesse sentido, o modelo de Koen et al. (2001), que posteriormente foi utilizado por Whitney (2007), traduz de forma clara a relação entre esses três elementos, enfatizando a interação entre eles. Koen et al. (2001) afirmam que o processo pode começar com a identificação de oportunidades ou com a geração de ideias, sendo que uma ideia necessita de uma ou mais oportunidades, bem como uma oportunidade pode necessitar de uma ou mais ideias.

A análise dos modelos evidenciou a relevância do alinhamento do FEl à estratégia da organização. Nesse sentido, ressalta-se o modelo de Khurana \& Rosenthal (1997), que destaca a importância do alinhamento entre o front end e a estratégia da organização. Em um sentido estratégico, a avaliação da situação de concorrência - varredura e análise ambiental - é mais uma atividade que precisa ser tratada durante o FEl (Kurkkio et al., 2011). Koen et al. (2001) incluem em seu modelo, por meio do que denominaram motor do front end, a liderança e cultura da organização. Diferentemente de Khurana \& Rosenthal (1997), Koen et al. (2001) incluem a estratégia como influenciador do processo, juntamente com as capacidades organizacionais, o mundo exterior (por exemplo, canais de distribuição, clientes e concorrentes) e a habilidade da ciência que será utilizada. Os fatores apresentados pelos trabalhos, podem, assim, ser agrupados no que poderia ser chamado de "direcionadores da inovação", a saber: estratégia, liderança, e cultura da organização. Considera-se que a estratégia engloba a análise de mercado e a análise tecnológica, como estabelecem Brem \& Voigt (2009).

Os modelos, seguindo uma tendência nos processos de inovação (Rothwell, 1994), apresentam a importância da integração entre as visões de mercado e da tecnologia, logicamente com graus diferentes em função de contextos específicos. Nesse sentido, destaque deve ser dado ao trabalho de Brem \& Voigt (2009), haja vista que os autores trabalharam justamente nessa relação a partir da teoria e de um estudo empírico em uma grande empresa de software e tecnologia da informação alemã. Toda essa discussão anterior leva a possibilidades de pesquisa futura, apresentadas a seguir.

\section{Perspectivas para trabalhos futuros}

A análise evidenciou que, apesar de algumas diferenças, os modelos apresentam pontos de convergência importantes. Esses pontos, em um primeiro momento, proporcionam um entendimento mais apurado do front end da inovação. Em um segundo momento, podem direcionar a organização e a estruturação do FEl em um contexto específico. Os pontos de convergência encontrados são os seguintes:

- Três elementos são recorrentes nos modelos de FEl: ideias, oportunidades e conceitos. Cada um desses elementos é gerido por um conjunto de atividades que, por sua vez, fazem uso de técnicas e ferramentas;

- 0 processo pode se iniciar tanto com uma ideia quanto com uma oportunidade; porém, o fim do FEl é um conceito inovador (um produto novo ou melhorado, um processo, um método de marketing ou um método organizacional);

- Os modelos a serem implementados são dependentes do contexto, ou seja, modelos específicos devem levar em conta o contexto no qual serão inseridos, incluindo os direcionadores da inovação (estratégia, liderança e cultura da organização);

- As contribuições advindas das visões sobre o mercado e a tecnologia devem ser levadas em conta, nesse sentido, tanto marketing quanto Pesquisa \& Desenvolvimento devem participar ativamente do FEl; 
- De um modo geral, os modelos não detalham nem a sequência e/ou relacionamento entre as atividades do FEl, nem os métodos e técnicas a serem utilizados nessas atividades, nem a escolha desses métodos e técnicas em função do contexto da organização. Porém as atividades do FEl são interativas e relacionadas, ou seja, o resultado de uma atividade pode demandar a realização de outra atividade, sendo essa já executada ou não. Nesse sentido, tem-se um processo interativo e recursivo.

Também foram encontrados, a partir da análise realizada, pontos de divergência ou que ainda necessitam de aprofundamentos teóricos e/ ou empíricos. A partir desses pontos, sugerem-se perspectivas para pesquisas.

Uma vez que os modelos encontrados não permitem a operacionalização de suas proposições, considera-se salutar aprofundar, a partir dos modelos existentes, questões relacionadas ao desenvolvimento de modelos específicos para o front end da inovação. A literatura, de um modo geral, considera importante a estruturação do front end da inovação. Entretanto, processos de aprendizado estão mais relacionados a sistemas flexiveis e que fomentem a experimentação. Uma vez que aprendizado leva a novos conhecimentos e esses são a base de inovações, principalmente as radicais (Aagaard \& Gertsen, 2011; Bertels et al., 2011), entende-se pertinente investigar até que ponto é adequada a estruturação formal do front end da inovação. Estudos sobre o relacionamento entre formalização e inovação têm produzido poucas e inconsistentes descobertas, o que resulta em baixo grau de generalização de resultados (Salmela et al., 2013). Questões que podem ser levantadas são: quais fatores influenciam o grau de formalização do FEl em um dado contexto? É mais adequado a organização possuir um conjunto de técnicas e ferramentas com sólida experiência no uso ou utilizar da experimentação e abrir possibilidades antes não conhecidas?

Partindo-se do fato de que a maioria dos modelos aborda a inovação em produtos (bens), considera-se importante aprofundar o estudo do FEl em outros contextos de desenvolvimento, tais como em serviços, processos, métodos de marketing e métodos organizacionais. Por exemplo, na inovação em processos, apesar da potencial redução de custos e ganho de eficiência com esse tipo de desenvolvimento, existe pouco conhecimento sobre como as empresas empregam seus esforços (Frishammar et al. 2011). Também considera-se relevante o estudo das interdependências entre os desenvolvimentos de tipos diferentes de inovação, como inovações em produtos (bens) e processos (Pruett \& Thomas, 2008).
Uma vez que alguns modelos apresentam apenas ideias e conceitos e outros apenas oportunidades e conceitos, sugere-se que pesquisas futuras procurem estabelecer até que ponto é adequado, nesse início do processo de inovação, diferenciar ou não ideias de oportunidades. Apesar de os conceitos de ideia e de oportunidade apresentados deixarem clara as diferenças entre eles, salienta-se que em algumas situações parece ser uma tarefa bastante complexa separá-los. Por exemplo, uma sugestão dada ao seu fornecedor por um grande consumidor industrial para um novo produto, contendo já algumas especificações preliminares, pode ser considerada uma ideia e ao mesmo tempo uma oportunidade? Até onde, do ponto de vista teórico, é interessante separar os dois conceitos? Do ponto de vista das organizações é útil essa separação? Essas são questões relevantes que podem direcionar os trabalhos futuros. As respostas a essas perguntas podem proporcionar uma base mais sólida para a estruturação e organização de processos específicos por meio da escolha de técnicas e ferramentas adequadas a ideias e oportunidades.

A partir da falta de consonância entre as atividades apresentadas na literatura para a gestão de oportunidades, ideias e conceitos, sugere-se que estudos futuros busquem um levantamento e a conceituação das atividades que podem ser executadas no front end da inovação. Esses estudos podem se concentrar em apenas um dos elementos, mas sugere-se que uma visão holística seja oferecida, haja vista o inter-relacionamento existente entre as atividades. Ainda nesse sentido, estudos podem levantar também as técnicas e ferramentas que podem auxiliar na execução dessas atividades, bem como critérios de comparação entre elas. Considera-se a identificação de critérios para a escolha de técnicas e ferramentas adequados ao front end da inovação, dado um contexto específico, um ponto relevante para novas pesquisas. Nesse sentido, que critérios devem ser levados em conta na escolha de técnicas e ferramentas a serem utilizadas? Quais fatores podem influenciar essa decisão?

Dado que um modelo de FEl deve ser adequado ao seu contexto, sugere-se que pesquisas futuras procurem aprofundar a relação entre o contexto e as atividades, técnicas e ferramentas relativas ao FEl. Assim, torna-se importante investigar formas de determinar a sequência de atividades adequadas bem como as técnicas e ferramentas a serem utilizadas com base no contexto da organização.

Uma vez que o FEl é um tema em desenvolvimento, com pouca abordagem empírica, trabalhos de campo que possibilitem vislumbrar como organizações específicas gerenciam esse início do processo da inovação podem, de um modo geral, contribuir 
para o entendimento do tema. Especificamente, trabalhos de natureza empírica podem proporcionar a possibilidade de comparar a sequência de atividades e a escolha de técnicas e ferramentas por gestores em diferentes setores de atuação.

\section{Conclusões}

0 presente trabalho analisou modelos de front end da inovação (FEl) a partir de um levantamento sistemático da literatura. 0 levantamento resultou em um portfólio de 268 artigos de periódicos, dos quais oito apresentam modelos de FEl relevantes para análise. Como resultado principal, elencou-se uma série de pressupostos obtidos a partir dos pontos convergentes entre os modelos analisados.

Uma vez que o FEl é um tema ainda carente de esforços tanto da academia quanto das organizações, pretende-se com o presente estudo melhorar o entendimento desse tema no escopo da gestão da inovação. Como propósito secundário espera-se também auxiliar no desenvolvimento de modelos mais aprofundados do ponto de vista de atividades, técnicas e ferramentas e, consequentemente, em um segundo momento, proporcionar a proposição de frameworks que possam melhorar a gestão desse início do processo de inovação, levando em consideração as especificidades organizacionais.

Conclui-se que embora os modelos identificados demonstrem uma contribuição ao processo de inovação, existem oportunidades de aprimoramento, destacadas pelas perspectivas de trabalhos futuros apresentadas neste artigo. Finalmente, espera-se que esses trabalhos possam contribuir para um entendimento cada vez mais aprofundado do front end da inovação, possibilitando melhorias não apenas nos conhecimentos teóricos sobre a área, mas também que possam auxiliar a melhorar a gestão no início dos processos de inovação, cujo resultado repercute em todo o processo.

\section{Referências}

Aagaard, A., \& Gertsen, F. (2011). Supporting radical front end innovation: perceived key factors of pharmaceutical innovation. Creativity \& Innovation Management, 20(4), 330-346. http://dx.doi.org/10.1111/j.14678691.2011.00609.x

Achiche, S., Appio, F. P., McAloone, T. C., \& Di Minin, A. (2013). Fuzzy decision support for tools selection in the core front end activities of new product development. Research in Engineering Design, 24(1), 1-18. http:// dx.doi.org/10.1007/s00163-012-0130-4

Alam, 1. (2006). Removing the fuzziness from the Fuzzy frontend of service innovations through customer interactions. Industrial Marketing Management, 35(4), 468-480. http://dx.doi.org/10.1016/j.indmarman.2005.04.004
Anderson, D. R., Sweeney, D. J., \& Williams, T. A. (1991). An introduction to management science: quantitative approaches to decision making (6th ed., pp. 6-7). USA: West Publishing. PMCid:PMC50736

Artto, K., Kulvik, l., Poskela, J., \& Turkulainen, V. (2011). The integrative role of the project management office in the front end of innovation. International Journal of Project Management, 29(4), 408-421. http://dx.doi. org/10.1016/j.jproman.2011.01.008

Backman, M., Börjesson, S., \& Setterberg, S. (2007). Working with concepts in the FuzzyFront end: exploring the context for innovation for different types of concepts at Volvo Cars. R\&D Management, 37(1), 17-28. http:// dx.doi.org/10.1111/j.1467-9310.2007.00455.x

Baregheh, A., Rowley, J., \& Sambrook, S. (2009). Towards a multidisciplinary definition of innovation. Management Decision, 47(8), 1323-1339. http://dx.doi. org/10.1108/00251740910984578

Bertels, H. M. J., Kleinschmidt, E. J., \& Koen, P. A. (2011). Communities of practice versus organizational climate: which one matters more to dispersed collaboration in the Front end of innovation? Journal of Product Innovation Management, 28(5), 757-772.

Bessant, J., Lamming, R., Noke, H., \& Phillips, W. (2005). Managing innovation beyond the steady state. Technovation, 25(12), 1366-1376. http://dx.doi. org/10.1016/j.technovation.2005.04.007

Boeddrich, H.-J. (2004). ldeas in the workplace: a new approach towards organizing the FuzzyFront end of the innovation process. Creativity \& Innovation Management, 13(4), 274-285. http://dx.doi.org/10.1111/ j.0963-1690.2004.00316.x

Brem, A., \& Voigt, K.-l. (2009). Integration of market pull and technology push in the corporate Front end and innovation management-insights from the German software industry. Technovation, 29(5), 351-367. http:// dx.doi.org/10.1016/j.technovation.2008.06.003

Brentani, U., \& Reid, S. E. (2012). The Fuzzy front-end of discontinuous innovation: lnsights for research and management. Journal of Product Innovation Management, 29(1), 70-87. http://dx.doi.org/10.1111/ j.1540-5885.2011.00879.x

Brolø, A. (2009). Innovative coopetition: the strength of strong ties. International Journal of Entrepreneurship and Small Business, 8(1), 110-134. http://dx.doi. org/10.1504/1JESB.2009.024108

Cagnazzo, L., Taticchi, P., \& Botarelli, M. (2008). A Literature review on innovation management tools. Revista de Administração da Universidade Federal de Santa Maria, 1(3), 316-330.

Clark, K. B., \& Wheelwright, S. C. (1993). Managing new product and process development: text and cases. New York: The Free Press.

Cooper, R. G. (1988). Predevelopment activities determine product success. Industrial Marketing Management, 17, 237-247. http://dx.doi.org/10.1016/00198501(88)90007-7

Cooper, R. G. (1993). Winning at new products: accelerating the process from idea to launch. New York: AddisonWesley.

Cooper, R. G. (2011). The innovation dilemma: how to innovate when the market is mature. Journal of Product Innovation Management, 28(1), 2-27. http://dx.doi. $\operatorname{org} / 10.1111 / j .1540-5885.2011 .00858 . x$ 
Cooper, R. G., \& Edgett, S. J. (2008). Maximizing productivity in product innovation. Research Technology Management, 51(2), 47-58.

Crawford, G. P., Broer, D. J., \& Bastiaansen, C. W. M. (2006). Engineering education on the 'Fuzzy Front end: a hightechnology entrepreneurship model. European Journal of Engineering Education, 31(2), 145-153. http://dx.doi. org/10.1080/03043790600566987

Crossan, M. M., \& Apaydin, M. (2010). A Multi-Dimensional Framework of Organizational innovation: a systematic review of the literature. Journal of Management Studies, 476), 1154-1191. http://dx.doi.org/10.1111/j.14676486.2009.00880.x

Flanagin, A. J. (2000). Social pressures on organizational website adoption. Human Communication Research, 26(4), 618-646. http://dx.doi.org/10.1111/j.1468-2958.2000. tb00771.x

Flint, D. J. (2002). Compressing new product success-tosuccess cycle time: deep customer value understanding and idea generation. Industrial Marketing Management, 31(4), 305-315. http://dx.doi.org/10.1016/S00198501(01)00165-1

Flynn, M., Dooley, L., O’Sullivan, D., \& Cormican, K. (2003). Idea management for organisational innovation. International Journal of Innovation Management, 74), 417-442. http://dx.doi.org/10.1142/S1363919603000878

Frishammar, J., Florén, H., \& Wincent, J. (2011). Beyond managing uncertainty: insights from studying equivocality in the Fuzzy Front end of product and process innovation projects. IEEE Transactions on Engineering Management, 58(3), 551-563. http://dx.doi. org/10.1109/TEM.2010.2095017

Garcia, R., \& Calatone, R. (2002). A critical look at technological innovation typology and innovativeness terminology: a literature review. Journal of Product Innovation Management, 19, 110-132. http://dx.doi. org/10.1016/S0737-6782(01)00132-1

Günzel, F., \& Holm, A. B. (2013). One size does not fit all - understanding the front-end and back-end of business model innovation. International Journal of Innovation Management, 17(1), 1-34. http://dx.doi. org/10.1142/S1363919613400021

Hammedi, W., Van Riel, A. C. R., \& Sasovova, Z. (2011). Antecedents and consequences of reflexivity in new product idea screening. Journal of Product Innovation Management, 28(5), 662-679.

Hannola, L., \& Ovaska, P. (2011). Challenging front-end-ofinnovation in information systems. Journal of Computer Information Systems, 52(1), 66-75.

Hennala, L., Parjanen, S., \& Uotila, T. (2011). Challenges of multi-actor involvement in the public sector front-end innovation processes: constructing an open innovation model for developing well-being services. European Journal of Innovation Management, 14(3), 364-387. http://dx.doi.org/10.1108/14601061111148843

Hüsig, S., \& Kohn, S. (2003). Factors influencing the Front end of the innovation process: a comprehensive review of selected empirical NPD and explorative FFE studies. In Proceedings of the 10th. International Product Development Management Conference, Brussels, Belgium.

Khurana, A., \& Rosenthal, S. R. (1997). Integrating the Fuzzy Front end of new product development. Sloan Management Review, 38(2), 103-120.

Khurana, A. \& Rosenthal, S. R. (1998). Towards holistic "Front ends" in new product development. Journal of
Product Innovation Management, 15(1), 57-74. http:// dx.doi.org/10.1016/S0737-6782(97)00066-0

Kim, J., \& Wilemon, D. (2002). Focusing the Fuzzy front-end in new product development. $R \& D$ Management, 32(4), 269-279. http://dx.doi.org/10.1111/1467-9310.00259

Koen, P. A., Ajamian, G. M., Boyce, S., Clamen, A., Fisher, E., Fountoulakis, S., Johnson, A., Puri, P., \& Seibert, R. (2002). Fuzzy Front end: effective methods, tools, and techniques. In P. Belliveau, A. Griffin \& S. Somermeyer (Eds.), The PDMA toolbook 1 for new product development (pp. 5-35). New York: John Wiley \& Sons Inc.

Koen, P. A., Ajamian, G., Burkart, R., Clamen, A., Davidson, J., D’Amore, R., Elkins, C., Herald, K., Incorvia, M., Johnson, A., Karol, R., Seibert, R., Slavejkov, A., \& Wagner, K. (2001). Providing clarity and a common language to the "Fuzzy Front end". Research Technology Management, 44(2), 46-55.

Kurkkio, M. (2011). Managing the Fuzzy front-end: insights from process firms. European Journal of Innovation Management, 14(2), 252-269. http://dx.doi. org/10.1108/14601061111124911

Kurkkio, M., Frishammar, J., \& Lichtenthaler, U. (2011). Where process development begins: a multiple case study of Front end activities in process firms. Technovation, 31(9), 490-504. http://dx.doi.org/10.1016/j. technovation.2011.05.004

Magnusson, P. R. (2009). Exploring the contributions of involving ordinary users in ideation of technology-based services. Journal of Product Innovation Management, 26(5), 578-593. http://dx.doi.org/10.1111/j.1540$5885.2009 .00684 x$

Martinsuo, M., \& Poskela, J. (2011). Use of evaluation criteria and innovation performance in the Front end of innovation. Journal of Product Innovation Management, 28(6), 896-914. http://dx.doi.org/10.1111/j.15405885.2011.00844.x

Meijer, I. S. M., Hekkert, M. P., \& Koppenjan, J. F. M. (2007). How perceived uncertainties influence transitions; the case of micro-CHP in the Netherlands. Technological Forecasting and Social Change, 74(4), 519-537. http:// dx.doi.org/10.1016/j.techfore.2006.02.007

Montoya-Weiss, M. M., \& 0'Driscoll, T. M. (2000). From experience: applying performance support technology in the Fuzzy Front end. Journal of Product Innovation Management, 17(2), 143-161. http://dx.doi.org/10.1016/ S0737-6782(99)00032-6

Muhdi, L., Daiber, M., Friesike, S., \& Boutellier, R. (2011). The crowdsourcing process: an intermediary mediated idea generation approach in the early phase of innovation. International Journal of Entrepreneurship \& Innovation Management, 14(4), 315-332.

Murphy, S. A., \& Kumar, V. (1997). The Front end of new product development: a Canadian survey. $R \& D$ Management, 27(1), 5-15. http://dx.doi. org/10.1111/1467-9310.00038

Nobelius, D., \& Trygg, L. (2002). Stop chasing the Front end process: management of the early phases in product development projects. International Journal of Project Management, 20(5), 331-3340. http://dx.doi. org/10.1016/S0263-7863(01)00030-8

Oliveira, M. G., \& Rozenfeld, H. (2010). Integrating technology roadmapping and portfolio management at the front-end of new product development. Technological Forecasting and Social Change, 77(8), 1339-1354. http://dx.doi. org/10.1016/j.techfore.2010.07.015 
Organização de Cooperação e Desenvolvimento Econômico - OECD. (2005). Oslo Manual: Guide-line for collecting and interpreting innovation data. (3rd ed.). European Comission: OECD. Retrieved from http://www. oecd.org

Paasi, J., Valkokari, P., Maijala, P., Luoma, T., \& Toivonen, S. (2007). Managing uncertainty in the front end of radical innovation development. In Proceeding of 16th International Conference on Management of Technology, Miami, FL, USA.

Perttula, M. K. (2004). Implications on cultural and formal processes of the front-end of new product development. In Proceedings of 2nd World Conference on Production and Operations Management, Cancun, Mexico.

Poskela, J., \& Martinsuo, M. (2009). Management control and strategic renewal in the Front end of innovation. Journal of Product Innovation Management, 26(6), 671-684. http://dx.doi.org/10.1111/j.1540-5885.2009.00692.x

Pruett, M. \& Thomas, H. (2008). Experience-based learning in innovation and production. $R \& D$ Management, 38(2), 141-153. http://dx.doi.org/10.1111/j.14679310.2008.00504.x

Quintane, E., Casselman, R. M., Reiche, B. S., \& Nylund, P. A. (2011). Innovation as a knowledge-based outcome. Journal of Knowledge Management, 15(6), 928-47. http://dx.doi.org/10.1108/13673271111179299

Reid, S. E., \& Brentani, U. (2004). The Fuzzy Front end of new product development for discontinuous innovations: a theoretical model. Journal of Product Innovation Management, 21(3), 170-184. http://dx.doi.org/10.1111/ j.0737-6782.2004.00068.x

Reinertsen, D. G. (1999). Taking the fuzziness out of the Fuzzy Front end. Research Technology Management, 42(6), 25-31.

Rothwell, R. (1994). Towards the fifth generation innovation process. International Marketing Review, 11(1). http:// dx.doi.org/10.1108/02651339410057491

Salmela, E., Santos, C., \& Happonen, A. (2013). Formalisation of front end innovation in supply network collaboration. International Journal of Innovation and Regional
Development, 5(1), 91-111. http://dx.doi.org/10.1504/ IJIRD.2013.052510

Sandmeier, P. (2009). Customer integration strategies for innovation projects: anticipation and brokering. International Journal of Technology Management, 48(1), 1-23. http://dx.doi.org/10.1504/IJTM.2009.024597

Smith, P.G., \& Reinertsen, D. G. (1991). Developing products in half the time. New York: Van Nostrand Reinhold.

Tidd, J., Bessant, J., \& Pavitt, K. (2008). Gestão da Inovação. (3rd ed.). Porto Alegre: Bookman. PMid:18344474 PMCid:PMC2270238

Toledo, J. C., Silva, S. L., Mendes, G. H. S., \& Jugend, D. (2008). Fatores críticos de sucesso no gerenciamento de projetos de desenvolvimento de produtos em empresas de base tecnológica de pequeno e médio porte. Gestão e Produção, 15(1), 117-134. http://dx.doi.org/10.1590/ S0104-530X2008000100011

Vandenbosch, B., Saatcioglu, A., \& Fay, S. (2006). Idea management: A systemic view. Journal of Management Studies, 43(2), 259-288. http://dx.doi.org/10.1111/ j.1467-6486.2006.00590.x

Whitney, D. E. (2007). Assemble a technology development toolkit. Research Technology Management, 50(5), 52-58.

Williams, M. A., Kochhar, A. K., \& Tennant, C. (2007). An object-oriented reference model of the Fuzzy Front end of the new product introduction process. International Journal of Advanced Manufacturing Technology, 34(78), 826-841. http://dx.doi.org/10.1007/s00170-006$0645-9$

Zomerdijk, L. G., \& Voss, C. A. (2011). NSD processes and practices in experiential services. Journal of Product Innovation Management, 28(1), 63-80. http://dx.doi. $\operatorname{org} / 10.1111 / j .1540-5885.2010 .00781 . x$

\section{Agradecimentos}

Os autores agradecem aos avaliadores da Revista Produção pela contribuição significativa para a melhoria do presente trabalho.

\title{
Front end of innovation models: similarities, differences and research perspectives
}

\begin{abstract}
The front end of innovation is the first sub-process of the innovation process. This sub-process includes the activities performed before the proposal and the adoption of a concept to be developed and implemented. This area has received little attention from academia and organizations; therefore, because organizations can substantially benefit from the optimization and improvement of the front end of innovation, the importance of studies that seek to understand the organization of this body of literature is justified. Thus, this paper aids in the development of theoretical concepts and fills the gaps observed in the existing research. In this sense, this paper reports the results of a study based on a survey and analyzes the literature related to the front end of innovation and, in particular, of this sub-process model. It also proposes a set of assumptions as the basis for the theoretical and empirical development of the subject. After a literature search was conducted in four electronic databases, 268 publications were identified, of which eight included front end of innovation models that were considered relevant for analysis. These models are compared based on some content analysis criteria of the articles. From this analysis, research perspectives are suggested, and points that require theoretical approaches are identified.
\end{abstract}

\section{Keywords}

Innovation. Front end of innovation. Innovation management. Literature review. 